\title{
Pimobendan in Chronic Right Heart Failure in a Left Ventricular Assist Device Patient
}

\author{
Maximilian Kreibich ${ }^{1} \quad$ Michael Berchtold-Herz ${ }^{1}$ \\ ${ }^{1}$ Department of Cardiovascular Surgery, University Heart Centre \\ Freiburg - Bad Krozingen, Freiburg, Germany \\ Thorac Cardiovasc Surg Rep 2016;5:39-40.
}

\author{
Friedhelm Beyersdorf ${ }^{1}$ Georg Trummer ${ }^{1}$
}

\author{
Abstract \\ Keywords \\ - circulatory assist \\ devices \\ - heart failure \\ - right ventricle
}

\author{
Address for correspondence Maximilian Kreibich, MD, Department of \\ Cardiovascular Surgery, University Heart Centre Freiburg - Bad \\ Krozingen, Hugstetter Str. 55, Freiburg 79106, Germany \\ (e-mail: maximilian.kreibich@uniklinik-freiburg.de).
}

\section{Introduction}

Chronic right heart failure in patients after left ventricular assist device (LVAD) implantation is an increasing problem with limited therapeutic options. ${ }^{1}$ When conservative heart failure medication to reduce right ventricular preand afterload is exhausted, positive inotropic drugs are the method of choice. ${ }^{1}$ However, inotropic therapy usually requires hospitalization of the patients. We report the case of a patient who developed chronic right heart failure 1 year after LVAD implantation. Initial recompensation was achieved, yet discharge was successful only after the offlabel use of the oral calcium sensitizer pimobendan. To the best of our knowledge, we hereby present the first case of the successful management of refractory right heart failure in a patient on prolonged LVAD therapy through oral pimobendan therapy.

\section{Case Report}

In this report, a 76-year-old male patient initially presented to our center in 2013 with ischemic cardiomyopathy with highly impaired left ventricular function (left ventricular ejection fraction [EF]: $20 \%$ and fractional shortening [FS]: $15 \%)$, moderate tricuspid regurgitation, and mildly impaired right ventricular function (tricuspid annular plane systolic excursion [TAPSE]: 14 mm). He was treated in December 2013 with a LVAD Heartmate II and a tricuspid valve annuloplasty with a 32-mm prosthetic ring (Carpentier-Edwards). The postoperative period was uneventful and the patient was discharged afterward. Regular follow-up checkups were organized in our LVAD outpatient clinic. Over the following period, the left ventricle was well unloaded and the LVAD function was uneventful. However, right heart function decreased steadily to a TAPSE of $7 \mathrm{~mm}$ despite increased sildenafil, oral diuretic therapy, and no signs of residual tricuspid regurgitation.

In October 2014, the patient routinely presented himself in our clinic, when a mild driveline infection was diagnosed. The patient was admitted and the local infection was successfully treated conservatively. Meanwhile, the patient suffered of recurrent pleural effusion and peripheral edema due to a highly impaired right heart function (TAPSE: $7 \mathrm{~mm}$ ) significantly limiting his level of physical activity. Treatment consisted of escalated diuretic and sildenafil therapy as well as chest drainage.

Over the next weeks, the patient's condition did not improve through conservative medical treatment and he had to be transmitted to our cardiac intensive care unit when he presented with worsening decompensated right heart failure. At that time, the patient's central venous saturation was 30\%. Echocardiographically, the right heart function was significantly impaired (TAPSE: $6-7 \mathrm{~mm}$ ). Pleural effusions and worse peripheral edema were visible. received

November 15, 2015

accepted after revision

December 14, 2015

published online

January 29, 2016
DOI http://dx.doi.org/

10.1055/s-0036-1571286. ISSN 2194-7635.
@ 2015 Georg Thieme Verlag KG
Stuttgart · New York

License terms

(®) $\Theta \circledast$ 
Recompensation was achieved through intravenous furosemide and inotropic therapy (dobutamine). Yet, successful catecholamine weaning was possible not before repeated intravenous levosimendan loading. At that point, all conservative medical heart failure medication including phosphodiesterase inhibitor therapy was exhausted, while levosimendan administration was only possible in an intensive care unit setting in our institution.

To allow the patient to be discharged from our center with refractory right heart failure, we decided on a therapeutic offlabel attempt with the oral calcium sensitizer pimobendan. The drug is approved in Japan for terminal heart failure but not within the European Union.

Informed consent was obtained and the patient's condition improved significantly over the next few weeks. We were able to discharge the patient with the oral pimobendan drug therapy ( $2.5 \mathrm{mg}$ twice a day) in due course with significantly improved right heart function. Pleural effusions were regressive and chest drainage was not necessary anymore. Right heart function was significantly improved echocardiographically before discharge (TAPSE: $15 \mathrm{~mm}$ ), while there was no difference in left ventricular function. The patient presented regularly in our LVAD clinic afterward at intervals of 4 weeks.

At every single presentation afterward from January to October 2015, no peripheral edema was visible and no pleural effusion was detectable. Echocardiographically, right heart function remained improved (TAPSE: $13-15 \mathrm{~mm}$ ), while the left ventricle did not present with better contractility (EF: $20-25 \%$ and FS: $10-20 \%$ ). The patient's general condition was significantly improved and he was able to fully participate in his every day live, limited only by his LVAD. There were no clinical signs of right heart failure present over a period of 10 months while the patient was taking pimobendan. Moreover, right heart function was considerably improved and remained comparably well while the patient was taking the calcium sensitizer.

\section{Discussion}

Pimobendan is a positive inotropic calcium sensitizer available in Japan functioning through a combination of cyclic adenosine monophosphate-dependent action and calciumsensitizing properties. ${ }^{2-4}$ In fact, the heart failure guideline in Japan has stated the necessity of investigating the role of oral inotropic agents in patients with chronic heart failure (CHF). ${ }^{2}$ Pimobendan, along with docarpamine and denopamine have proven to significantly improve the New York Heart Association functional class, cardiothoracic ratio, and B-type natriuretic peptide levels in elderly patients with $\mathrm{CHF}^{2}$ through enhance contractility, relaxation, and improved cardiac performance. ${ }^{5,6}$ Moreover, the number of emergency room visits and hospitalizations were significantly reduced in patients taking the oral calcium sensitizers. ${ }^{2,7}$ These results were confirmed in another study showing that pimobendan in conjunction with conventional therapy for heart failure decreases the readmission rate in elderly patients with severe heart failure for at least 2 years. ${ }^{8}$

There is only one case report about the use of pimobendan in the setting of a cardiac assist device therapy: Imamura et al were able to successfully prevent the implantation of a right ventricular assist device in a patient with right ventricular dysfunction who was implanted a LVAD through pimobendan and sildenafil therapy. ${ }^{9}$

To the best of our knowledge, we hereby present the first case of the successful management of refractory right heart failure in a patient on prolonged LVAD therapy through oral pimobendan therapy in a dosage of $2.5 \mathrm{mg}$ twice a day. Due to the good experience with the drug, we believe that further research on the therapeutic effects of positive inotropic calcium sensitizers is necessary in patients with right heart failure especially in patients with ongoing LVAD therapy.

\section{References}

1 Argiriou M, Kolokotron SM, Sakellaridis T, et al. Right heart failure post left ventricular assist device implantation. J Thorac Dis 2014;6 (Suppl 1):S52-S59

2 Murai K, Seino Y, Kimata N, et al. Efficacy and limitations of oral inotropic agents for the treatment of chronic heart failure. Int Heart J 2013;54(2):75-81

3 Fujino K, Sperelakis N, Solaro RJ. Sensitization of dog and guinea pig heart myofilaments to $\mathrm{Ca} 2+$ activation and the inotropic effect of pimobendan: comparison with milrinone. Circ Res 1988;63(5): 911-922

4 van Meel JC, Entzeroth M, Redemann N, Haigh RM. Effects of pimobendan and its metabolite on myofibrillar calcium responsiveness and ATPase activity in the presence of inorganic phosphate. Arzneimittelforschung 1995;45(2):136-141

5 Remme WJ, Wiesfeld AC, Look MP, Kruyssen HA. Hemodynamic effects of intravenous pimobendan in patients with left ventricular dysfunction. J Cardiovasc Pharmacol 1989;14(Suppl 2):S41-S44

6 Permanetter B, Sebening H, Hartmann F, Klein G. Acute effects of intravenous UD-CG 115 BS (pimobendan) on the cardiovascular system and left ventricular pump function. J Cardiovasc Pharmacol 1989;14(Suppl 2):S36-S40

7 Kubo SH, Gollub S, Bourge R, et al; The Pimobendan Multicenter Research Group. Beneficial effects of pimobendan on exercise tolerance and quality of life in patients with heart failure. Results of a multicenter trial. Circulation 1992;85(3):942-949

8 Kawano H, Arakawa S, Satoh O, et al. Effect of pimobendan in addition to standard therapy for heart failure on prevention of readmission in elderly patients with severe chronic heart failure. Geriatr Gerontol Int 2014;14(1):109-114

9 Imamura T, Kinugawa K, Nitta D, et al. Biventricular failure with low pulmonary vascular resistance was managed by left ventricular assist device alone without right-sided mechanical support. J Artif Organs 2015;18(3):272-275 